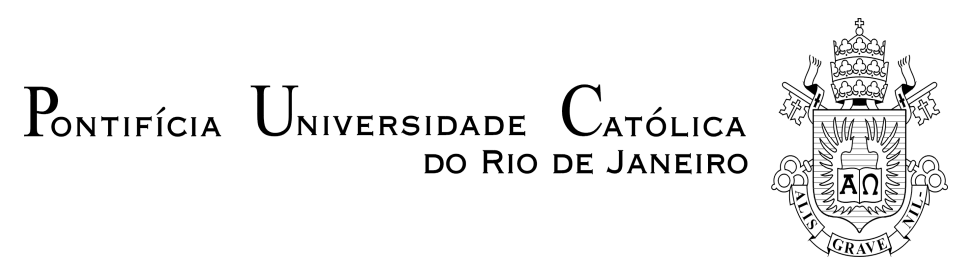

José Enrique Gutiérrez Ramírez

\title{
Variabilidade Espacial do Parâmetro Geomecânico RQD no Depósito Mineral Animas-Peru
}

\section{Dissertação de Mestrado}

Dissertação apresentada como requisito parcial para obtenção do título de Mestre pelo Programa de PósGraduação em Engenharia Civil da PUC-Rio.

Orientador: Prof. Celso Romanel 


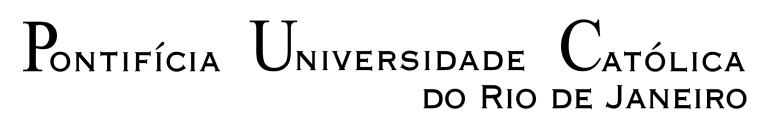

DO RIO DE JANEIRO

José Enrique Gutiérrez Ramírez

\title{
Variabilidade Espacial do Parâmetro Geomecânico RQD no Depósito Mineral Animas-Peru
}

\begin{abstract}
Dissertação apresentada como requisito parcial para obtenção do grau de Mestre pelo Programa de PósGraduação em Engenharia Civil da PUC-Rio. Aprovada pela Comissão Examinadora abaixo assinada.
\end{abstract}

Prof. Celso Romanel Orientador

Departamento de Engenharia Civil - PUC-Rio

Prof. Sergio Augusto Barreto da Fontoura Departamento de Engenharia Civil - PUC-Rio

Prof. Franklin dos Santos Antunes Departamento de Engenharia Civil - PUC-Rio

Prof. Fernando Saboya Albuquerque Jr Laboratório de Engenharia Civil - UENF

Prof. José Eugênio Leal Coordenador Setorial do Centro Técnico Científico da PUC-Rio

Rio de Janeiro, 27 de Março de 2009. 
Todos os direitos reservados. É proibida a reprodução total ou parcial do trabalho sem autorização da universidade, do autor e do orientador.

\section{José Enrique Gutiérrez Ramírez}

Graduou-se em engenharia na Universidade Nacional de Engenharia (UNI), em Lima - Peru, no curso de Engenharia Geológica. Trabalhou em empresas de mineração na área de modelagem geológica e avaliação de jazidas, com aplicação de métodos geoestatísticos.

Ficha Catalográfica

Gutiérrez Ramírez, José Enrique
Variabilidade espacial do parâmetro geomêcanico RQD no
depósito Animas - Peru / José Enrique Gutiérrez Ramírez;
orientador: Celso Romanel - Rio de Janeiro: PUC,
Departamento de Engenharia Civil, 2009.
v.,167 f. : il. ; $29.7 \mathrm{~cm}$.
Dissertação (Mestrado em Engenharia Civil)-Pontifícia
Universidade Católica do Rio de Janeiro, Rio de Janeiro,
2009.
Inclui referências bibliográficas.
1. Engenharia civil - Tese. 2. Krigagem ordinária. 3.
Modelagem 3D. 4. Simulação seqüencial Gaussiana. 5.
Modelo de blocos. I. Romanel, Celso. II. Pontifícia
Universidade Católica do Rio de Janeiro. Departamento de
Engenharia Civil. III. Título.

CDD: 624 


\section{Agradecimentos}

Ao professor Celso Romanel, pela orientação e amizade conquistada.

À minha noiva, Yajaira, agradecido pelo carinho, amor e companheirismo, em cada dia desses dois longos anos.

Aos meus pais, agradeço por todo o carinho e o incentivo que sempre me proporcionaram, incondicionalmente.

À minha avó Rosa, a quem sempre tive no meu coração.

Ao Departamento de Engenharia Civil da PUC-RIO pela infraestrutura e ensinamentos.

À FAPERJ e à PUC-Rio, pelo apoio financeiro.

À mineradora BATEAS SAC pela cessão dos dados utilizados nesse estudo, em especial "a colaboração dos engenheiros Enrique Velarde e Arturo Salvador.

À Deus, criador de todas as coisas, fonte de fé e esperança. 


\section{Resumo}

Gutíerrez Ramírez, José Enrique. Romanel, Celso (Orientador). Variabilidade Espacial do Parâmetro Geomêcanico RQD no Depósito Mineral Animas-Peru. Rio de Janeiro, 2009. 167p. Dissertação de Mestrado - Departamento de Civil, Pontifícia Universidade Católica do Rio de Janeiro.

Esta dissertação desenvolve um estudo sobre a aplicação das técnicas de modelagem geológica e análises geoestatísticas na avaliação da variabilidade espacial do parâmetro geotécnico (RQD) no depósito Animas - Peru. O estudo das cinco litologias presentes no depósito permitirá melhorar o conhecimento da qualidade do maciço rochoso em regiões não-amostradas. Nesta pesquisa foram empregados o método da krigagem ordinária, para estimação da variabilidade espacial, e a simulação seqüencial Gaussiana, para simulação da distribuição dos valores de RQD. Ambos os resultados foram comparados com um técnica de estimativa clássica - o método do inverso do quadrado da distância. Verificou-se que os resultados provenientes do método clássico e da krigagem ordinária são bastante semelhantes entre si, enquanto que valores produzidos pela simulação Gaussiana apresentaram acentuadas diferenças em todas as litologias analisadas. No estudo foram utilizados os programas computacionais Datamine, para modelagem geológica, e Isatis v.7 para análises geoestatísticas.

\section{Palavras-chave}

Krigagem ordinária. Modelagem 3D. Simulação sequencial Gaussiana. Modelo de blocos. 


\section{Abstract}

Gutíerrez Ramírez, José Enrique. Romanel Celso (advisor). Spatial Variability of the Geomechanical Parameter RQD at the Ore Deposit Animas - Peru. Rio de Janeiro, 2009. 167p. M.Sc. Dissertation Departamento de Engenharia Civil, Pontifícia Universidade Católica do Rio de Janeiro.

This thesis investigates the application of geological modeling techniques and geostatistical methods for estimation of the spatial variability of the geotechnical parameter RQD at the ore deposit Animas - Peru. The study of five different rock layers will permit the improvement of the overall knowledge about the quality of the rock mass, mainly in the regions where the existence of experimental samples is limited or not available at all. In this research the ordinary kriging method was used for studies of spatial variability and the Gaussian sequential simulation for analysis of the distribution of RQD values throughout the rock mass. The results of both methods were compared with those calculated on basis of a classical estimation technique - the method of the inverse of the squared distances. It was concluded that the values obtained by ordinary kriging and the classical method are quite similar between themselves but the results with Gaussian simulation show significant differences in all the rock layers investigated. In this research the following computational programs were used: Datamine, for the geologic modeling, and Isatis v.7, for geostatiscal analyses.

\section{Keywords}

Ordinary kriging. 3D modeling. Gaussian sequential simulation. Block model. 


\section{Sumário}

1. INTRODUÇÃO 17

1.1 Parâmetro RQD 19

$\begin{array}{ll}1.2 \text { Variabilidade espacial } & 21\end{array}$

$\begin{array}{ll}1.3 \text { Objetivos e estrutura da dissertação } & 23\end{array}$

2. LOCALIZAÇÃO, FISIOGRAFIA E GEOLOGIA 24

2.1. Localização 24

2.2. Relevo e cllima 26

2.3. Geologia 25

2.3.1. Formação Sencca 26

2.3.2. Grupo Tacaza 26

2.3.3. Depósitos quaternários $\quad 27$

2.3.4. Geologia estrutural 28

2.3.5. Gênese e tipo do depósito mineral 28

3. METODOLOGIAS ESTATÍSTICAS E GEOESTATÍSTICAS 32

3.1. Análise estatística 32

3.1.1. Estatística descritiva 32

3.1.2. Modelos de distribuição de probabilidades 36

3.2. Análise geoestatística 38

3.2.1. Funções aleatórias estacionárias 38

3.2.2. Analises da variabilidade espacial 39

3.2.3. Estimativas clássicas 53

3.2.4. Estimativas geoestatísticas $\quad 55$

3.2.5. Incertezas geoestatísticas 63

3.2.6. Simulações geoestatísticas 65

4. MODELAGEM 72

4.1. Banco de dados $\quad 73$

4.2. Modelagem geométrica e amostragem $\quad 74$

4.2.1. Metodologia da amostragem do parâmetro RQD 74

4.2.2. Critérios da modelagem geológica 79

4.3. Modelagem numérica $\quad 81$

4.3.1. Malhas de blocos

4.3.2. Regularização 83

4.3.3. Modelagem numérica 86

4.3.4. Validação cruzada $\quad 92$

4.3.5. Métricas para análises comparativas 94

5 APRESENTAÇÃO E ANÁLISE DOS RESULTADOS 96

5.1. Modelagem geológica da área 96

5.1.1. Modelagem geométrica 101 
5.1.2. Modelagem numérica 108

5.2. Estatística básica 115

5.2.1. Estatística descritiva $\quad 115$

5.2.2. Estatísticas das amostras regularizadas originais e desagregadas $\quad 115$

5.2.3. Transformação a uma variável normalmente distribuída por litologia 118

5.3. Variografia 119

5.3.1. Determinação dos variogramas experimentais por litologia $\quad 119$

5.3.2. Modelagem dos variogramas experimentais por litologia 121

5.4. Validação cruzada e vizinhança de estimativa 137

$\begin{array}{ll}\text { 5.4.1. Validação cruzada dos dados originais } & 137\end{array}$

5.4.2. Validação cruzada dos dados transformados 143

5.5. Análises dos resultados do parâmetro RQD por IQD, OK e SGS 149

5.5.1. Estimativa do RQD pelo método IQD 149

5.5.2. Estimativa do RQD pelo método OK 150

5.5.3. Estimativa do RQD pelo método SGS 150

$\begin{array}{ll}\text { 5.5.4. Comparação dos resultados } & 151\end{array}$

6 CONCLUSÕES E SUGESTÕES 159

REFERÊNCIAS BIBLIOGRÁFICAS 162 


\section{Lista de figuras}

Figura 1-1 Modelagem geológica - numérica de formações geológicas ...................23

Figura 2-1 Localização da área de estudo. ............................................................. 24

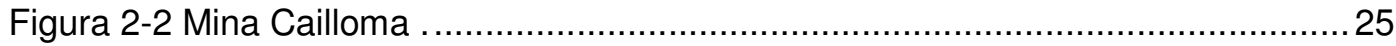

Figura 2-3 Mapa geológico da mina Cailloma. ................................................... 27

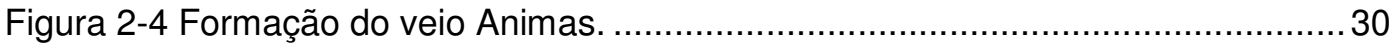

Figura 2-5 Formação da caldeira Cailloma. ...................................................... 30

Figura 2-6 Mapa dos veios da mina Cailloma. .................................................. 31

Figura 3-1 Comportamento das medidas de correlação $\tilde{C}(h), \tilde{\rho}(h), \tilde{\gamma}(h)$. ................. 42

Figura 3-2 Esquema básico de uma função variograma. ........................................ 43

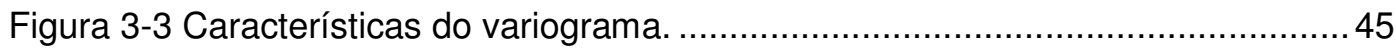

Figura 3-4 Tipos de anisotropía variográfica; a) geométrica; b) zonal. ....................... 46

Figura 3-5 Modelos variográficos com patamar. ................................................. 47

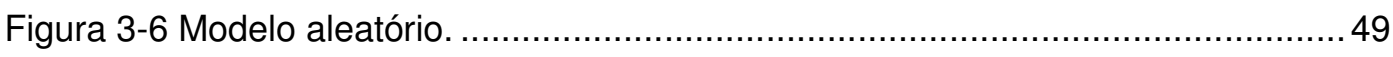

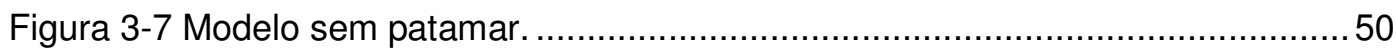

Figura 3-8 Dessegregação em células ( Isaaks \& Srivastava, 1989)...........................52

Figura 3-9 Potência $\beta$ do método IQD (Villanueva, 2000)......................................54

Figura 3-10 Método da poligonal a) mediatrizes; b) bissetrizes...............................55

Figura 3-11 Avaliação de um bloco sub-dividido em 4 sub-blocos ............................63

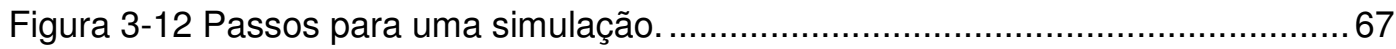

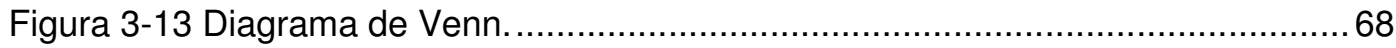

Figura 4-1 Fluxo da modelagem (modificado a partir de S. Houlding, 1994)..............73

Figura 4-2 Medição de parâmetros geológicos - geotécnicos nas sondagens.............76

Figura 4-3 Testemunhos serrados longitudinalmente........................................... 76

Figura 4-4 Procedimentos utilizados para a determinação do RQD ...........................78

Figura 4-5 Método dos perfis ou das seções (Yamamoto, 1996) ...............................80

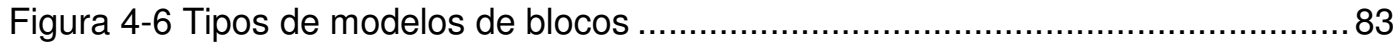

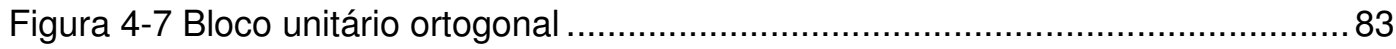

Figura 4-8 Regularização de uma sondagem (Journel \& Huijbregts, 1989)..................84

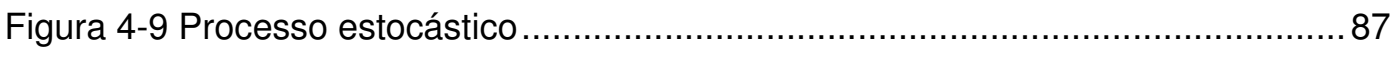

Figura 4-10 Processo de estimativa através de Krigagem ..................................... 88

Figura 4-11 Processo de simulação geoestatística - SGS ....................................... 89

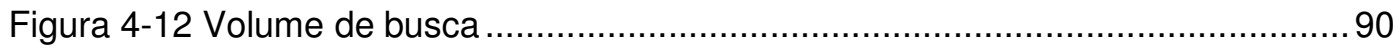

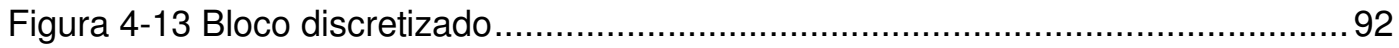




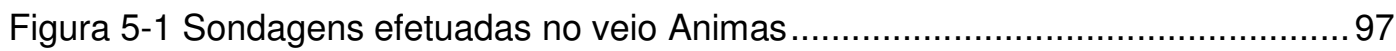

Figura 5-2 Vista vertical (Leste-Oeste) das sondagens efetuadas no veio Animas .....98

Figura 5-3 Vista vertical (Norte-Sul) das sondagens efetuadas no veio Animas...........98

Figura 5-4 Niveis 7,8 e 9 do veio Animas................................................................ 99

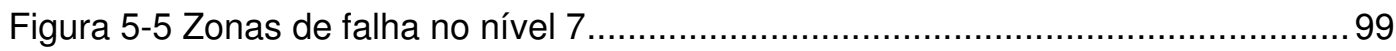

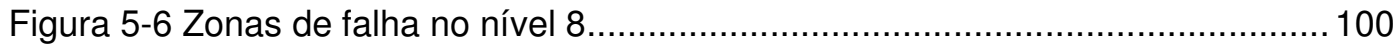

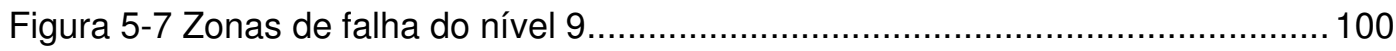

Figura 5-8 Topografia 3D da área de produção ................................................... 104

Figura 5-9 Modelo geológico AGV ............................................................. 105

Figura 5-10 Modelo geológico ANDPORF ...................................................... 105

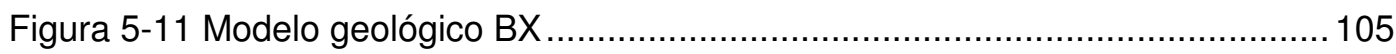

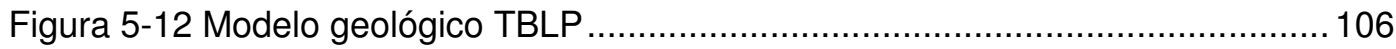

Figura 5-13 Modelo geológico das zonas de falha do veio Animas ......................... 106

Figura 5-14 Modelo geológico $V$ e suas zonas de falha ....................................... 107

Figura 5-15 Conjunto de modelos geológicos .................................................. 107

Figura 5-16 Amostras regularizadas da litologia AGV ......................................... 109

Figura 5-17 Amostras regularizadas da litologia ANDPORF ................................ 109

Figura 5-18 Amostras regularizadas da litologia BX .......................................... 110

Figura 5-19 Amostras regularizadas da litologia TBLP.........................................110

Figura 5-20 Amostras regularizadas da litologia $V$.............................................. 110

Figura 5-21 Malha de blocos da litologia AGV ................................................ 112

Figura 5-22 Malha de blocos da litologia ANDPORF....................................... 113

Figura 5-23 Malha de blocos da litologia BX ................................................... 113

Figura 5-24 Malha de blocos da litologia TBLP ................................................. 114

Figura 5-25 Malha de blocos da litologia V ...................................................... 114

Figura 5-26 Histograma das amostras da litologia AGV .....................................116

Figura 5-27 Histograma das amostras da litologia ANDPORF ...............................116

Figura 5-28 Histograma das amostras da litologia BX........................................ 117

Figura 5-29 Histograma das amostras da litologia TBLP .................................... 117

Figura 5-30 Histograma das amostras da litologia $\mathrm{V}$............................................ 118

Figura 5-31 Rotação de eixos - variograma direcional ...................................... 121

Figura 5-32 Variograma omnidirecional da litologia AGV ..................................... 122

Figura 5-33 Variograma omnidirecional da litologia ANDPORF ............................. 122

Figura 5-34 Variograma omnidirecional da litologia BX........................................ 123

Figura 5-35 Variograma omnidirecional da litologia TBLP ...................................... 123

Figura 5-36 Variograma omnidirecional da litologia $\mathrm{V}$.......................................... 124

Figura 5-37 Variograma omnidirecional da litologia AGV .................................... 125 
Figura 5-38 Variograma omnidirecional da litologia ANDPORF ............................ 125

Figura 5-39 Variograma omnidirecional da litologia BX..................................... 126

Figura 5-40 Variograma omnidirecional da litologia TBLP..................................... 126

Figura 5-41 Variograma omnidirecional da litologia $\mathrm{V}$.............................................. 127

Figura 5-42 Variograma na direção 1 da litologia ANDPORF.................................128

Figura 5-43 Variograma na direção 2 da litologia ANDPORF.................................. 128

Figura 5-44 Variograma na direção 3 da litologia ANDPORF..................................129

Figura 5-45 Variograma na direção 1 da litologia TBLP ........................................ 129

Figura 5-46 Variograma na direção 2 da litologia TBLP ...................................... 130

Figura 5-47 Variograma na direção 3 da litologia TBLP ...................................... 130

Figura 5-48 Variograma na direção 1 da litologia $V$.............................................. 131

Figura 5-49 Variograma na direção 2 da litologia $V$.............................................. 131

Figura 5-50 Variograma na direção 3 da litologia $V$............................................. 132

Figura 5-51 Variograma na direção 1 da litologia ANDPORF................................ 133

Figura 5-52 Variograma na direção 2 da litologia ANDPORF.................................. 133

Figura 5-53 Variograma na direção 3 da litologia ANDPORF................................... 134

Figura 5-54 Variograma na direção 1 da litologia TBLP ...................................... 134

Figura 5-55 Variograma na direção 2 da litologia TBLP ........................................ 135

Figura 5-56 Variograma na direção 3 da litologia TBLP ......................................... 135

Figura 5-57 Variograma na direção 1 da litologia $V$.............................................. 136

Figura 5-58 Variograma na direção 2 da litologia $V$............................................. 136

Figura 5-59 Variograma na direção 3 da litologia $\bigvee$........................................... 137

Figura 5-60 Validação cruzada das amostras da litologia AGV .............................. 139

Figura 5-61 Validação cruzada das amostras da litologia ANDPORF ...................... 140

Figura 5-62 Validação cruzada das amostras da litologia BX............................... 141

Figura 5-63 Validação cruzada das amostras da litologia TBLP ............................. 142

Figura 5-64 Validação cruzada das amostras da litologia V ................................... 143

Figura 5-65 Validação cruzada das amostras transformadas (AGV) ........................145

Figura 5-66 Validação cruzada das amostras transformados (ANDPORF) ............... 146

Figura 5-67 Validação cruzada das amostras transformados (BX)......................... 147

Figura 5-68 Validação cruzada das amostras transformados (TBLP) .......................148

Figura 5-69 Validação cruzada das amostras transformados (V)...........................149

Figura 5-70 Distribuição do RQD na litologia AGV ................................................ 153

Figura 5-71 Distribuição do RQD na litologia ANDPORF ....................................... 154

Figura 5-72 Distribuição do RQD na litologia BX.............................................. 154

Figura 5-73 Distribuição do RQD na litologia TBLP ............................................ 155

Figura 5-74 Distribuição do RQD na litologia V .................................................. 155 


\section{Lista de Tabelas}

Tabela 1-1 Programas para modelagem geológica - geoestatística ............................. 24

Tabela 4-1 Classificação do índice RQD ........................................................... 78

Tabela 5-1 Campos da base de dados da mineradora Bateas ......................................96

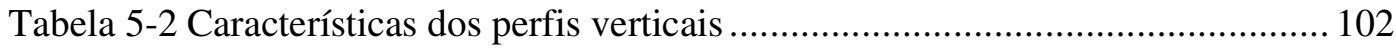

Tabela 5-3 Características dos perfis horizontais .................................................. 103

Tabela 5-4 Número de amostras por códigos litológicos....................................... 103

Tabela 5-5 Número de amostras regularizadas por código litológico ........................ 108

Tabela 5-6 Características do modelo da litologia AGV ......................................... 111

Tabela 5-7 Características do modelo da litologia ANDPORF ............................... 111

Tabela 5-8 Características do modelo da litologia BX .......................................... 111

Tabela 5-9 Características do modelo da litologia TBLP ..................................... 112

Tabela 5-10 Características do modelo da litologia V .............................................. 112

Tabela 5-11 Estatística descritiva das amostras regularizadas ............................... 115

Tabela 5-12 Comparação entre valores originais e estimativas Gaussianas ................. 119

Tabela 5-13 Parâmetros utilizados na determinação do variograma experimental ...... 119

Tabela 5-14 Parâmetros utilizados na determinação dos variogramas direcionais ...... 120

Tabela 5-15 Atitude dos variogramas direcionais ............................................. 120

Tabela 5-16 Modelos variográficos omnidirecionais - dados originais..................... 121

Tabela 5-17 Modelos variográficos omnidirecionais - dados transformados. .............. 124

Tabela 5-18 Modelos variográficos direcionais - dados originais ............................ 127

Tabela 5-19 Modelos variográficos direcionais - dados transformados .................... 132

Tabela 5-20 Volume de busca por código litológico ................................................. 137

Tabela 5-21 Características das amostras utilizadas na estimativa (OK-IQD) ............. 138

Tabela 5-22 Número de sub-blocos por código litológico ...................................... 138

Tabela 5-23 Coeficiente de correlação por código litológico .................................... 138

Tabela 5-24 Estatística do erro reduzido (ER) por código litológico .......................... 138

Tabela 5-25 Volume de busca por código litológico ............................................ 144

Tabela 5-26 Características do número de amostras na estimativa de KS.................. 144

Tabela 5-27 Número de sub-blocos por código litológico....................................... 144 
Tabela 5-28 Coeficiente de correlação por código litológico .................................... 144

Tabela 5-29 Estatística do erro reduzido (ER) por código litológico ........................... 144

Tabela 5-30 Estatística descritiva do RQD estimados pelo método IQD ..................... 150

Tabela 5-31 Estatística descritiva do RQD estimados com o método OK .................. 150

Tabela 5-32 Estatística do RQD simulados pelo SGS (50 simulações) ..................... 150

Tabela 5-33 Estatística do RQD simulados pelo SGS (100 simulações).................... 151

Tabela 5-34 MAPE com o RQD obtido pela OK ................................................. 152

Tabela 5-35 MAPE modificando os modelos variográficos no método SGS .............. 152

Tabela 5-36 MAPE modificando a vizinhança no método SGS ............................. 152 


\section{Lista de símbolos}

\section{Caracteres latinos}

a - Amplitude variográfica nos modelos de variograma

A - Amplitude dos dados na análise estatística

C - Patamar dos modelos variográficos

Cov - Covariância

${ }_{A A}$ - Covariância entre os pontos na área A

$C_{U V}$ - Covariância entre as variáveis $U$ e $V$

$C(h)$ - Função covariância

$\mathrm{C}_{0} \quad$ - Efeito pepita nos modelos variográficos

Cov - Covariância espacial entre duas variáveis

CV - Coeficiente de variação

$d_{i} \quad$ - Distancia do centro do bloco à amostra

DG - Desvio geométrico

$E\{V(x)\} \quad$ - Valor esperado da variável $V$

$f(x)$ - Função densidade de probabilidade

$h$ - Distância entre dois pontos de amostragem 
$k \quad$ - Coeficiente percentílico de curtose

$m$ - Média aritmética das amostras

M - Mediana de um conjunto de dados

MG - Média geométrica

n - Número de dados nas análises estatísticas

$p$ - Fator de ponderação em técnica de dessegregação.

Pr - Probabilidade de ocorrência de um evento

$Q_{n} \quad$ - Quartis de uma distribuição

$R\left(x_{0}\right)$ - Erro da estimação

$s_{i} \quad$ - Área poligonal

$v_{1}, \ldots, v_{n}-$ Valores amostrados

$V \quad$ - Estimativas da variável regionalizada $\mathrm{V}$

$\hat{V} \quad$ - Valor estimado da variável $\mathrm{V}$

$\tilde{V}_{A}-$ Valor estimado da área $\mathrm{A}$

$\tilde{V}_{\text {dados }}(x)$ - Valor estimado com krigagem simples

$\tilde{V}_{\text {sim }}$ - Valor simulado condicionado 


\author{
$\tilde{V}_{\text {ucsim }}$ - Valor simulado não-condicionado \\ Var - Variância \\ X - Variável contínua \\ $w_{i} \quad$ - Fatores de ponderação
}

\title{
Caracteres gregos
}

$\lambda \quad$ - Valor esperado de uma distribuição

$\lambda_{0} \quad$ - Fator de ajuste da estimativa

$\sigma \quad$ - Desvio padrão populacional

$\sigma^{2} \quad$ - Variância populacional

$\sigma_{\text {ок }}{ }^{2}$ - Variância da krigagem Ordinaria

$\sigma_{R}{ }^{2}$ - Variância dos erros da krigagem

$\sigma_{\mathrm{sK}}^{2}$ - Variância da krigagem simples

$\varphi\left(\mathrm{A}\right.$, Íc $_{\mathrm{c}}$ - Distribuição espacial de probabilidades

$\mu \quad$ - Média aritmética populacional no modelo de distribuição normal

$\mu_{1}, \mu_{2}$ - Multiplicadores de Lagrange

$\rho(h)$ - correlograma 
$\gamma(h)$ - Função variograma

$\gamma_{U V}(h)$ - Função variograma cruzado das variáveis $U$ e $V$

\& - Valor proporcional constante

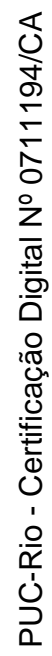

